\title{
Haemorrhage in the lens: spontaneous occurrence in congenital cataract
}

\author{
KAZUHIKO UNOKI, KUMIKO NAKAO, AND NORIO OHBA \\ From the Department of Ophthalmology, Kagoshima University Faculty of Medicine, Kagoshima-shi, Japan
}

SUMmaRY A 2-month-old female infant had bilateral congenital cataract. In the left eye a blood clot was situated on the subcapsular polar cataract. An uneventful lensectomy combined with anterior vitrectomy by the pars plicata approach revealed remnants of the posterior portion of hyaloid artery on the optic nerve head. It is likely that the spontaneous and long-lasting haemorrhage in the lens had been caused by rupture of the anterior end of the hyaloid artery attached to the posterior lens surface and had occurred in the late prenatal or early postnatal period.

Haemorrhage in the crystalline lens is very uncommon.' Occasional cases have been caused by rupture of the lens capsule allowing the blood to enter the lens stroma, as complications of intraocular surgery, ${ }^{2}$ or from perforating ocular injury. ${ }^{3} \mathrm{We}$ report the case of a 2-month-old patient who had a spontaneous and long-lasting haemorrhage in the lens associated with congenital posterior polar cataract.

\section{Case report}

A female infant was born with birth weight of $3840 \mathrm{~g}$ after a full-term, normal, spontaneous delivery. The mother had a history of rubella infection at the age of 12 years and had no problems during the pregnancy. There was no contributory family history or parental consanguinity. Two weeks after the birth the mother had noticed a whitish appearance in the infant's pupils. At the age of 2 months the infant was referred to us for investigations of bilateral congenital cataract.

Physical examinations revealed that the infant had developed well and had no systemic anomalies. Radiological examinations, including skull CT scan, showed normal appearances. The results of the following laboratory studies were also normal: erythrocyte count, packed cell volume, leucocyte count, platelet count, bleeding and coagulation time of the blood, serological tests for viral infections, chromosomal analysis of the leucocyte.

Correspondence to Kazuhiko Unoki, MD, Department of Ophthalmology, Kagoshima University Faculty of Medicine, Usuki-cho 1208-1, Kagoshima-shi, Japan (890).
On ocular examination the infant was found to have poor fixation on objects. There was no abnormality in the ocular adnexas and outer segments. The corneas were clear and measured $8.5 \mathrm{~mm}$ in diameter. The anterior chambers were clear and normally deep, with well developed angle structures. The pupils were round and reacted well to light or mydriatics. The lenses had prominent opacities, which were judged to inhibit visual development. They were treated surgically as described below. Applanation tonometry gave $10 \mathrm{mmHg}$ in both eyes. The electroretinograms, ocular ultrasonograms, and orbital CT scans gave normal results.

\section{RIGHT EYE}

The lens was normal in position, shape, and size. The anterior portion of the lens was clear, but there was a dense, circular opacity in the subcapsular cortex of the posterior pole. There was neither haemorrhage nor aberrant vessel in the lens. The vitreous underlying the posterior polar cataract was invisible. The peripheral region of the vitreous and fundus visible through the clear peripheral lens was normal.

Because the infant's fixation remained poor, an aspiration of the lens material by the limbal approach was performed on the 130th day of life. Although most of the lens could be easily aspirated, the dense posterior polar opacity was so calcareous and firmly adherent to the posterior lens capsule that it had to be left, and it was later dissected during cataract surgery of the fellow eye. As a result, the pupillary area became clear, and ophthalmoscopy revealed normal vitreous and fundus. 


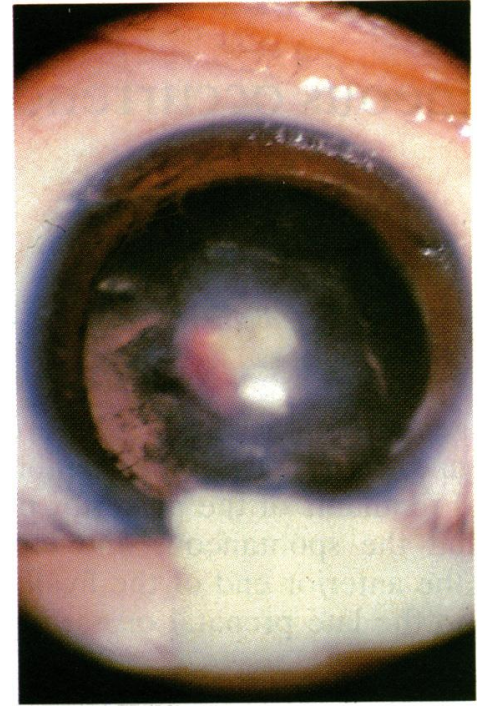

Fig. 1 Deposit of clotted blood on the posterior polar cataract.

LEFT EYE

Several thread-like remnants of pupillary membrane were attached to the iris only. The lens was normal in position, shape, and size. There was a circular dense opacity, situated in the subcapsular cortical region of the posterior pole and surrounded by a larger but weaker opacity. On the anterior surface of the temporal border of the central dense opacity was deposited a rectangular, sharply-delimited haemorrhage, about $2 \times 1 \mathrm{~mm}$, which appeared clotted and must have persisted for a long time. No vascular components were detectable adjacent to the haemorrhage. Because of this dense opacity the lens capsule in the posterior pole and the underlying vitreous could not be examined (Fig. 1). The whole anterior cortex, embryonal nucleus, and peripheral portion of the posterior cortex were clear, permitting us to visualise normal peripheral vitreous and fundus.

During observations over two months after the initial visit the clotted haemorrhage in the lens remained unchanged. Lensectomy combined with a shallow anterior vitrectomy was carried out on the 165th day of life by the pars plicata approach: the clotted haemorrhage was easily aspirated together with the neighbouring lens materials. A part of the calcareous cataract adhered to the posterior lens capsule was excised and aspirated en bloc with the anterior vitreous; no bleeding occurred, nor were any aberrant vascular or fibrous tissues present. Light microscopic examinations of aspirated materials revealed lens capsule, lens epithelium, and normal and degenerated lens fibres intermingled with clotted blood, but no vascular or fibrous tissues.

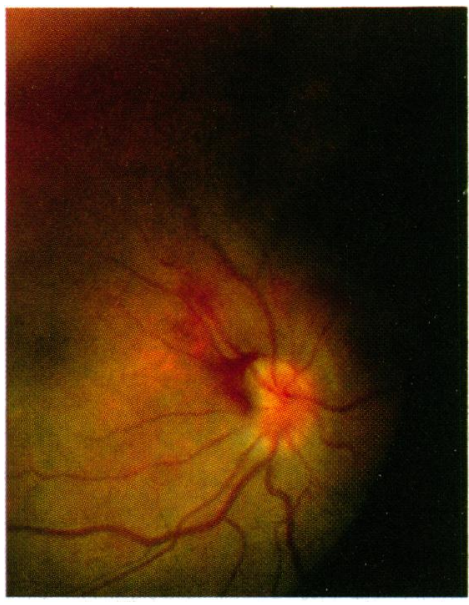

Fig. 2 Postoperative haemorrhage on the optic nerve head projecting into the vitreous cavity from the persistent hyaloid artery.

The postoperative course was uneventful, and the pupillary area became clear enough for us to examine the vitreous and fundus. On the second postoperative day ophthalmoscopy revealed linear fresh haemorrhages on the optic nerve head; projecting a short distance into the vitreous cavity, and on the neighbouring retina (Fig. 2). These haemorrhages cleared completely in three weeks and were followed by retention of a fine strand devoid of blood originating from the surface of the optic disc. Eventually the infant learned a stable, good central fixation by wearing aphakic correction glasses.

\section{Discussion}

Haemorrhage in the lens as a solitary condition is very rare. Previous reports described cases in which the haemorrhage in the lens occurred as complications of intraocular surgery, such as a filtration operation or peripheral iridectomy for glaucoma, ${ }^{2}$ or as sequelae of perforating ocular injury. ${ }^{3}$ These complications must have been caused by rupture of the lens capsule, in particular a part of the anterior capsule, allowing blood from the anterior chamber to enter the lens stroma.

It is noticeable that the infant reported on here had an apparently spontaneous haemorrhage in the lens in the sense that it was not subject to either surgical or traumatic intervention. Stein ${ }^{4}$ briefly reported a similar case: a 4-year-old child with unilateral congenital cataract and convergent strabismus had small haemorrhage under the posterior lens capsule, which remained unchanged for a year. 
A common clinical feature in these two cases of spontaneous haemorrhage in the lens is a deposit of clotted blood in the congenital posterior polar cataract. In addition to the brief note by Stein ${ }^{4}$ the present case may offer some clues for understanding the pathogenesis of the haemorrhage in the lens. The right eye had neither haemorrhage in the lens nor overt ocular anomaly. By contrast the left eye, with its haemorrhage in the lens, had remnants of pupillary membrane and, moreover, persistent posterior end of hyaloid artery on the optic disc projecting a short distance into the vitreous cavity. These observations indicate an overall delay of regression of the fetal vascular systems round the lens and vitreous, ${ }^{5}$ and they also suggest that the anterior end of the hyaloid artery is persistent. It may therefore be possible to assume that a rupture of the anterior end of the hyaloid artery attached to the posterior lens surface caused bleeding into the lens. The incident must have occurred in the late prenatal period, or in the early postnatal period, since the haemorrhage in the lens was clotted, and the anterior end of the hyaloid artery had regressed two months after birth.

Haemorrhage in the lens must remain for a long time. For the management of a congenital cataract associated with it the pars plicata approach for lensectomy combined with anterior vitrectomy may be better than the conventional corneal approach, since firm adherence of the posterior lens capsule to the anterior vitreous membrane is to be expected. In the present case fresh haemorrhages from the persistent hyaloid artery occurred on the optic disc in the early postoperative days. A similar complication may also occur spontaneously. ${ }^{6}$

\section{References}

1 Duke-Elder S. Diseases of the lens. General consideration. In: Duke-Elder S, ed. System of ophthalmology. London: Kimpton, 1969; 12: 8.

2 Stevens PR, Chatterjee S. Haemorrhage into the lens. A complication of intra-ocular surgery. Br J Ophthalmol 1959; 43: 42-5.

3 Masuda Y, Koide M. Hemorrhage into the cataractous lens. Report of a case. Jpn J Clin Ophthalmol 1971; 25: 1755-8. 4 Stein R. Haemorrhage in the lens. Acta Med Orient 1955; 14: 209.

5 Duke-Elder S. Anomalies of the vascular system. In: Duke-Elder S, ed. System of ophthalmology. London: Kimpton, 1964; 3: $764-82$.

6 Tolentino FI, Schepens CL, Freeman HM. Vitreoretinal disorders. Diagnosis and management. Philadelphia: Mosby, 1976: 191-3.

Accepted for publication 29 November 1985. 